\section{ADEQUATE DISTRIBUTION OF WARM CARDIOPLEGIC SOLUTION}

Seventy-five patients undergoing coronary artery bypass grafting were randomized to receive warm antegrade $(N=25)$, warm retrograde $(N=25)$, or a combination of warm antegrade and retrograde $(N=25)$ delivery of blood cardioplegic solution. Myocardial oxygen utilization, lactate and acid metabolism, and adenine nucleotides and their degradation products were measured during the operation and cardiac function was assessed postoperatively. Warm retrograde delivery of cardioplegic solution increased lactate and acid release during cardioplegia and reperfusion, decreased left ventricular adenosine triphosphate concentrations, and reduced the washout of adenine nucleotide degradation products from both left and right ventricles. Warm antegrade delivery of cardioplegic solution resulted in less lactate and acid release during cardioplegia but more lactate accumulated in the territory of the left anterior descending artery during the crossclamp period. Intermittent antegrade delivery of the cardioplegic solution during combination cardioplegia washed out lactate and acid, which suggested inhomogeneous delivery of the cardioplegic solution during continuous retrograde cardioplegia. Combination cardioplegia best preserved adenosine triphosphate in the left ventricle and resulted in the best postoperative left and right ventricular function. A combination of intermittent antegrade and continuous retrograde delivery of cardioplegic solution provided better myocardial protection than either antegrade or retrograde delivery of cardioplegic solution alone. (J THORAC CARDIOvasC SURG 1995;110:800-12)

Nobuhiko Hayashida, MD, John S. Ikonomidis, MD,*

Richard D. Weisel, MD, ${ }^{* *}$ Toshizumi Shirai, MD, Joan Ivanov, RN, Susan Carson, AHT, Molly K. Mohabeer, BSc, Laura C. Tumiati, BSc, and Donald A. G. Mickle, MD, Toronto, Ontario, Canada
$\mathrm{N}^{\mathrm{o}}$ ormothermic blood cardioplegia offers the promise to resuscitate the ischemic myocardium and reduce the morbidity and mortality for patients

From the Division of Cardiovascular Surgery, Department of Clinical Biochemistry, and the Center for Cardiovascular Research, The Toronto Hospital and the University of Toronto, Toronto, Ontario, Canada.

Presented at the Sixty-sixth Scientific Sessions of the American Heart Association, Atlanta, Ga., Nov. 9, 1993.

Supported by the Medical Research Council of Canada (grant MT9829) and the Heart and Stroke Foundation of Ontario (grant B2267).

Received for publication May 17, 1994.

Accepted for publication Dec. 20, 1994.

Address for reprints: Richard D. Weisel, MD, The Toronto Hospital, 200 Elizabeth St., EN 14-215, Toronto, Ontario M56 2C4, Canada

*Fellow of the Medical Research Council of Canada.

${ }^{*}$ Career Investigator of the Heart and Stroke Foundation of Ontario.

Copyright (C) 1995 by Mosby-Year Book, Inc.

$0022-5223 / 95 \$ 5.00+0 \quad 12 / 1 / 63011$ with high-risk disease. Warm antegrade delivery of cardioplegic solution maintains myocardial oxygen consumption and reduces anaerobic lactate production, provided the flow rate is greater than 80 $\mathrm{ml} / \mathrm{min}$ and the hemoglobin level is greater than 80 $\mathrm{gm} / \mathrm{L}^{1}$ However, coronary occlusions, particularly of the left anterior descending artery, limit perfusion of cardioplegic solution when an arterial conduit is used to bypass this artery. ${ }^{2}$ Retrograde cardioplegia permits delivery of cardioplegic solution to the region of the left anterior descending artery despite a coronary occlusion, ${ }^{2}$ but may provide limited perfusion to the posterior septum and right ventricle. $^{3}$ Therefore a combination of antegrade and retrograde delivery of cardioplegic solution may be required to provide adequate myocardial perfusion during warm blood cardioplegia.

This prospective randomized clinical trial was performed in patients at low risk for adverse clinical outcomes to document differences of myocardial metabolism and ventricular function. We previously demonstrated that patients undergoing elective op- 
Table I. Clinical data

\begin{tabular}{lcccc}
\hline & Antegrade & Combination & Retrograde & $p$ Value \\
\hline No. of patients & 25 & 25 & 25 & 0.99 \\
Age (yr) & $58 \pm 2$ & $58 \pm 2$ & $58 \pm 2$ & 0.77 \\
Gender (M/F) & $24 / 1$ & $24 / 1$ & $23 / 2$ & 0.44 \\
NYHA class (II/III/IV) & $3 / 13 / 9$ & $0 / 14 / 11$ & $1 / 13 / 11$ & 0.93 \\
Left main disease (Y/N) & $2 / 23$ & $4 / 21$ & $4 / 21$ & 0.48 \\
LVEF (>0.6/0.4-0.6/<0.4) & $12 / 10 / 3$ & $9 / 11 / 5$ & $11 / 13 / 1$ & 0.29 \\
Urgent operation & 4 & 8 & 4 & 0.31 \\
Grafts (2/3/4/5) & $3 / 5 / 12 / 5$ & $0 / 4 / 18 / 3$ & $2 / 8 / 13 / 2$ & 0.06 \\
XCL time (min) & $65 \pm 3$ & $69 \pm 3$ & $59 \pm 3$ & 0.07 \\
CPB time (min) & $98 \pm 3$ & $97 \pm 3$ & $87 \pm 4$ & \\
\hline
\end{tabular}

Ages and times are given as the mean value plus or minus the standard error of the mean. The $p$ values refer to the results of analysis of variance for continuous variables and $\chi^{2}$ or Fisher's exact test for categoric variables. $M$, Male; $F$, female; $N Y H A$, New York Heart Association; $Y$, yes; $N$, no; $L V E F$, left ventricular ejection fraction; $X C L$, crossclamp.

erations who received blood rather than crystalloid cardioplegia had improved metabolic recovery and better postoperative ventricular function. ${ }^{4} \mathrm{We}$ were then able to demonstrate that blood cardioplegia reduced the risks of urgent operations for unstable angina by reducing perioperative morbidity and mortality. ${ }^{5}$ Therefore in this prospective, clinical trial we evaluated myocardial metabolism and ventricular function to determine whether subtle differences could be found. The finding of a significant benefit with combination cardioplegia would allow us to anticipate performance of a large randomized, clinical trial in patients with high-risk conditions to determine whether this cardioplegic technique will reduce morbidity and mortality.

\section{Methods}

Patient population. Seventy-five patients scheduled for isolated coronary artery bypass grafting by one surgeon agreed to participate in this study of alternative cardioplegic techniques. All patients signed a consent form approved by the human experimentation committee. Patients admitted to the study were 36 to 77 years old, had double- or triple-vessel coronary artery disease, and had adequate preoperative ventricular function (left ventricular ejection fraction greater than $30 \%$ by preoperative single-plane contrast ventriculography). Table I describes the clinical data of patients in this study.

Operative technique. Cardiopulmonary bypass (CPB) was established with a single two-stage right atrial cannula. During bypass, the hematocrit value was maintained between $20 \%$ and $25 \%$, pump flows between 2.0 and 2.5 $\mathrm{L} / \mathrm{min}$ per square meter, and mean arterial pressure between 50 and $60 \mathrm{~mm} \mathrm{Hg}$, with administration of sodium nitroprusside or phenylephrine hydrochloride as required. Patients were not actively cooled but their systemic temperatures were allowed to drift to $33^{\circ} \pm 1^{\circ} \mathrm{C}$. Rewarming was begun during construction of the last three anastomoses. A left internal mammary artery was anastomosed to the left anterior descending coronary artery as the last graft in all patients.
Cardioplegia groups. Patients were randomly assigned, by means of computer-generated randomization table, to one of three cardioplegic strategies (Table I and Fig. 1).

Blood cardioplegic solution was prepared by mixing four parts of oxygenated blood to each part of crystalloid solution ${ }^{1}$ and was delivered by the Buckberg-Shiley Plus system (Shiley Inc., Irvine, Calif.). In all patients, cardiac arrest was achieved with an antegrade infusion of $500 \mathrm{ml}$ high-potassium cardioplegic solution (containing 27 $\mathrm{mEq} / \mathrm{L}$ of potassium) delivered into the aortic root at $37^{\circ} \mathrm{C}$ and at a pressure of $70 \mathrm{~mm} \mathrm{Hg}$ measured through a separate port of the solution cannula (for antegrade delivery of cardioplegic solution, DLP Inc., Grand Rapids, Mich.; for antegrade/retrograde delivery of cardioplegic solution, Research Medical Inc., Midvale, Utah).

Antegrade delivery of cardioplegic solution (antegrade cardioplegia; $N=25$ ). After cardiac arrest, low-potassium cardioplegic solution (containing $13 \mathrm{mEq} / \mathrm{L}$ potassium) at $37^{\circ} \mathrm{C}$ was delivered into the aortic root at a flow rate of $200 \mathrm{ml} / \mathrm{min}$. Flow of the cardioplegic solution was interrupted, and the aortic root vented, whenever necessary to permit adequate visualization during the critical portion of distal anastomosis construction. After the completion of each distal anastomosis, the vein grafts were attached to a manifold system to allow delivery of cardioplegic solution into the aortic root and all completed vein grafts. During subsequent distal anastomoses cardioplegic solution was delivered into vein grafts with the aortic root vented. A "catch-up" retrograde infusion was given after each interruption in a volume that was calculated by the perfusionist to precisely compensate for the period of cessation of flow and to maintain the average cardioplegic solution flow rate near $150 \mathrm{ml} / \mathrm{min}$.

Retrograde delivery of blood cardioplegic solution (retrograde cardioplegia; $N=25$ ). After cardiac arrest was achieved with the initial antegrade infusion, the aortic root was vented and retrograde delivery of cardioplegic solution was begun via the coronary sinus cannula (Research Medical Inc.) at a flow rate of $200 \mathrm{ml} / \mathrm{min}$. Coronary sinus pressure was monitored continuously by the separate pressure monitoring line and maintained at less than $40 \mathrm{~mm} \mathrm{Hg}$ throughout the procedure. Distal and proximal anastomoses were constructed in an alternating manner. As in the antegrade group, flow of the cardio- 


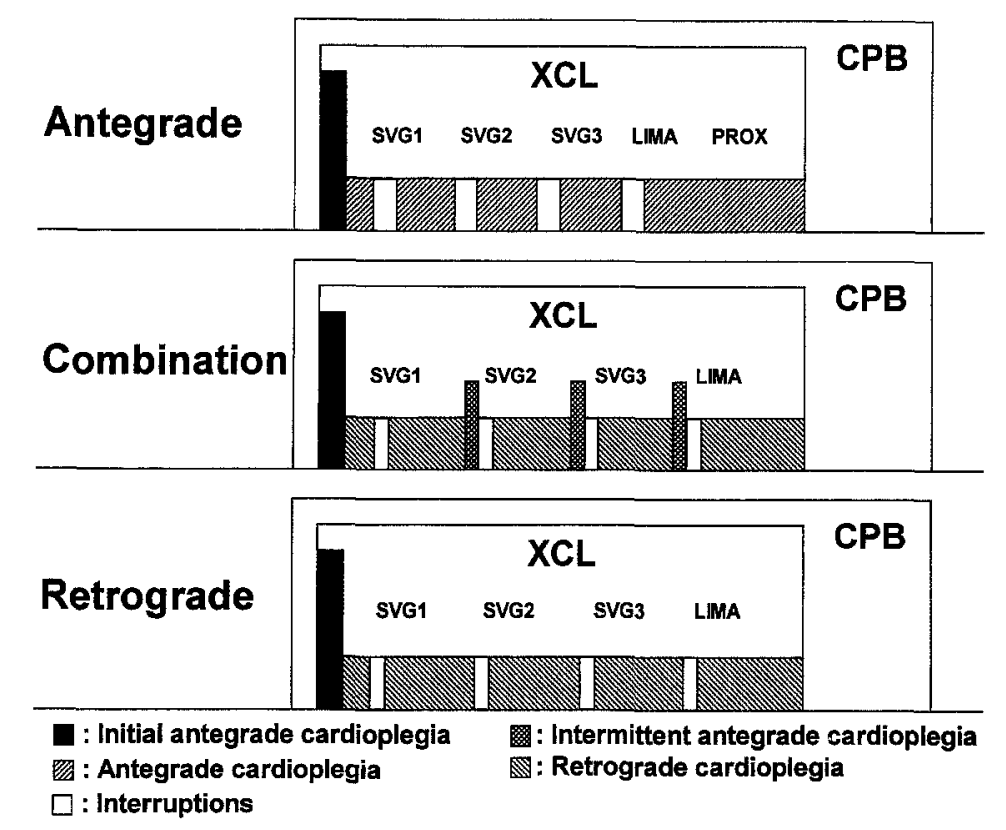

Fig. 1. Cardioplegic techniques are depicted. In the combination and retrograde groups, proximal anastomoses were constructed after each distal anastomosis during retrograde infusions of cardioplegic solution. LIMA, Anastomoses of left internal mammary artery; $S V G$, anastomoses of saphenous vein grafts; $P R O X$, proximal anastomoses of saphenous vein grafts; $X C L$, aortic crossclamp.

plegic solution was interrupted whenever necessary to achieve adequate visualization during construction of distal anastomoses. A "catch-up" infusion was given to maintain the average flow rate near $200 \mathrm{ml} / \mathrm{min} .^{6}$

Combination of antegrade and retrograde delivery of cardioplegic solution (combination cardioplegia; $N=25$ ). Cardioplegic solution was given in a manner identical to that of warm retrograde cardioplegia. A "catch-up" retrograde infusion was given after each interruption for the construction of a distal anastomosis. In addition, antegrade intermittent infusions of cardioplegic solution (250 $\mathrm{ml}$ ) were given through the aortic root at a flow rate sufficient to maintain aortic root pressure at $50 \mathrm{~mm} \mathrm{Hg}$ after completion of each proximal anastomosis. Infusion of the cardioplegic solution was never done simultaneously in an antegrade and retrograde manner.

The three cardioplegic techniques are illustrated in Fig. 1.

Oxygen, lactate, and pH assays. Arterial and coronary sinus blood samples were obtained simultaneously during bypass before application of the crossclamp, immediately after crossclamp release, 10 minutes after crossclamp removal, and 10 minutes after $\mathrm{CPB}$ was discontinued. To uncover any latent anaerobic metabolism, arterial and coronary sinus samples were also taken after 5 minutes of atrial pacing at a rate of 110 beats/min during bypass 10 minutes after crossclamp release and 10 minutes after discontinuation of CPB.

During crossclamping, cardioplegic solution and coronary venous blood samples were obtained every $10 \mathrm{~min}$ utes in the antegrade group and immediately before the completion of proximal anastomoses during retrograde delivery of cardioplegic solution in both the retrograde group and the combination group. Additional blood samples were taken 30 seconds after the initiation of intermittent antegrade delivery of cardioplegic solution in the combination group. Coronary venous samples were taken from the coronary sinus during antegrade cardioplegic solution delivery and from the aortic root during retrograde cardioplegic solution delivery.

Blood samples were assayed for the partial pressure of oxygen $\left(\mathrm{PO}_{2}\right)$ and carbon dioxide, $\mathrm{pH}$ (Acid-Base Laboratory, Radiometer, Copenhagen, Denmark), and oxygen saturation (Co-Oximeter, Instrumentation Laboratory Inc., Lexington, Mass.). Oxygen content ( $\mathrm{O}_{2} \mathrm{Con}$ ) was calculated from the formula

$$
\mathrm{O}_{2} \mathrm{Con}=1.39 \mathrm{Hgb} \times \mathrm{S}+0.0031 \mathrm{PO}_{2}
$$

where $\mathrm{Hgb}$ is the hemoglobin concentration and $\mathrm{S}$ is the oxygen saturation. Myocardial oxygen extraction was calculated as the arterial or cardioplegic solution oxygen content minus the coronary venous oxygen content. Measurements were made at $37^{\circ} \mathrm{C}$ and corrected to the myocardial temperature at the time of sampling, which was measured in the region of the left anterior descending artery with a temperature probe..$^{1,6,7}$

Blood samples for lactate concentration were mixed with a measured volume of $6 \%$ perchloric acid. Lactate concentration was measured in the protein-free supernatant by an enzymatic method (Rapid Lactate Stat Pack kit; Calbiochem-Behring, La Jolla, Calif.). Myocardial lactate extraction was calculated in the same manner as oxygen extraction. Negative lactate extraction was expressed as lactate production. 
The concentration of hydrogen ion $\left(\left[\mathrm{H}^{+}\right]\right)$in each blood sample was determined by converting the measured $\mathrm{pH}$ value to $\left[\mathrm{H}^{+}\right]$by the formula $\left[\mathrm{H}^{+}\right]=\operatorname{antilog}(-\mathrm{pH})$. Measurements were made at $37^{\circ} \mathrm{C}$ and corrected to the myocardial temperature at the time of sampling. Myocardial acid production was calculated as the coronary venous effluent $\left[\mathrm{H}^{+}\right]$minus the arterial or cardioplegic solution $\left[\mathrm{H}^{+}\right]$.

During cardioplegic arrest myocardial consumption of oxygen, lactate release, and acid release were calculated as cardioplegic solution flow multiplied by the difference between the arterial and coronary sinus content. ${ }^{1,7}$

Myocardial adenine nucleotides, degradation products, and lactate concentration. Transmural left and right ventricular biopsy specimens were obtained with a Tru-Cut biopsy needle (Travenol Labs, Deerfield, Ill.) from the territory of the left anterior descending coronary artery and right ventricular free wall to determine myocardial concentration of adenosine triphosphate (ATP), adenosine diphosphate (ADP), adenosine monophosphate (AMP), and total adenine nucleotide degradation products $($ TDP $=$ adenosine + inosine + hypoxanthine + xanthine) in 36 patients (12 patients in each cardioplegia group). Myocardial lactate concentrations were measured in 39 patients (13 patients in each cardioplegia group). The computer-generated randomization table allocated patients to have biopsy studies allocated to adenine nucleotides or lactate on a random basis. The specimens were flash-frozen in liquid nitrogen and then freeze dried. Myocardial adenine nucleotides and degradation products were measured by high-performance liquid chromatography, with a modification of the step gradient technique described by Hull-Ryde and associates. ${ }^{8}$ Myocardial lactate concentrations were measured by spectrophotometric techniques (Greiner Selective Analyzer II, Greiner Electronics, Langenthal, Switzerland; Perkin-Elmer 650-150 fluorescence spectrometer, Perkin-Elmer Corp., Norwalk, Conn.). ${ }^{9}$ Biopsy samples were taken at $37^{\circ} \mathrm{C}$ during $\mathrm{CPB}$ before crossclamp application, immediately after release of the crossclamp, and again after 10 minutes of reperfusion.

Hemodynamic measurements. Heart rate (HR), mean arterial blood pressure (MAP), mean pulmonary artery pressure (MPA), mean right atrial pressure (RAP), and pulmonary capillary wedge pressure (PCWP) were measured. Cardiac output (CO) was measured with the thermodilution technique. Derived hemodynamic indices were calculated as follows: cardiac index $(\mathrm{CI})=\mathrm{CO} /$ body surface area (liters per minute per square meter); stroke index $(\mathrm{SI})=\mathrm{CI} / \mathrm{HR}$ (milliliters per minute per square meter); left ventricular stroke work index $=$ SI $\times($ MAP PCWP) $\times 0.0136$ (grams - meter per square meter); right ventricular stroke work index $=\mathrm{SI} \times(\mathrm{MPA}-\mathrm{RAP}) \times$ 0.0136 (grams $\cdot$ meter per square meter). These hemodynamic variables were measured before initiation of CPB and at 10 minutes, 1 hour, 4 hours, 12 hours, and 24 hours after cessation of CPB. The relationships between left and right ventricular stroke work indices and the corresponding atrial pressures were evaluated by an analysis of covariance. Postoperative volume repletion followed a standard protocol and was done by persons who
Table II. Clinical outcomes

\begin{tabular}{lrccc}
\hline & Antegrade & Combination & Retrograde & $p$ Value \\
\hline No. of patients & 25 & 25 & 25 & \\
Deaths & 0 & 1 & 2 & 0.36 \\
MI & 0 & 3 & 1 & 0.16 \\
LOS & 1 & 3 & 2 & 0.59 \\
IABP & 1 & 1 & 2 & 0.77 \\
Stroke & 1 & 0 & 0 & 0.37 \\
\hline
\end{tabular}

The $p$ values refer to the results of analysis of variance for continuous variables and $\chi^{2}$ or Fisher's exact test for categoric variables. $M I$, Myocardial infarction; $L O S$, low output syndrome; $L A B P$, intraaortic balloon pump assistance.

were unaware of the intraoperative cardioplegic technique used.

Creatine kinase. An antibody inhibition technique was used to measure the MB isozyme of creatine kinase (CK-MB). The sequential CK-MB measurements were done $2,4,8,16,24$, and 48 hours after crossclamp release. Integration of the area under the concentration-time curve for CK-MB allowed calculation of the total CK-MB release, expressed as units times hours per liter. ${ }^{1,6,7}$

Statistical analysis. Statistical analysis was done with the SAS program (SAS Institute, Cary, N.C.). Analysis of variance was used to test the effect of cardioplegic strategies on myocardial oxygen utilization, lactate and acid metabolism, concentration of adenine nucleotides, degradation products and myocardial lactate, hemodynamic variables, and postoperative CK-MB release. When analysis of variance indicated a significant effect of cardioplegic group or time $(p<0.05)$, the differences were specified with Duncan's multiple range test. Continuous variables are listed as the mean and standard error of the mean. Categoric data are displayed as the absolute and percent frequency, and were analyzed by $\chi^{2}$ or Fisher's exact tests where appropriate. Statistical significance was assumed at a probability level of less than 0.05 .

For the hemodynamic data an analysis of covariance was needed to compare the three cardioplegic groups for the relation between left or right ventricular stroke work index or cardiac index and pulmonary capillary wedge pressure.

\section{Results}

Operative variables. Table I indicates that the number of bypass grafts was not different among cardioplegic groups $(p=0.31)$. However, the retrograde groups tended to have fewer bypasses and shorter crossclamp $(p=0.06)$ and bypass $(p=0.07)$ times. When these times were corrected by the number of grafts, the crossclamp time per graft ( $p=$ $0.47)$ and the CPB time per graft $(p=0.20)$ were not different among groups.

Clinical outcomes. The clinical outcomes are shown in Table II. Two of the patients in the retrograde cardioplegia group and one of the patients in the combination cardioplegia group died in 
this series of 75 patients (4\%). Two patients with diffuse triple-vessel disease who received retrograde cardioplegia died of heart failure despite balloon pump and inotropic support. One patient who received combination cardioplegia required an extensive bowel resection because of an ischemic bowel syndrome 11 days after bypass operation and died of renal failure 83 days after operation. Four patients had a perioperative myocardial infarction (defined as either new $Q$ waves or ischemic ST segment changes with a concomitant rise in CK-MB levels). Six patients had low output syndrome (defined as the requirement for an intraaortic balloon pump or inotropic support for more than 30 minutes despite optimization of preload and afterload). Intraaortic balloon pump support was required postoperatively in four patients. The prevalence of these events was not statistically different between groups.

Six patients required inotropic medication and four of these six patients required intraaortic balloon pumping after operation because of transient ventricular dysfunction in the operating room. In the intensive care unit only one of the six patients had a low left ventricular stroke work index $\left(9 \mathrm{gm} \cdot \mathrm{m} / \mathrm{m}^{2}\right)$ at an elevated pulmonary artery wedge pressure $(19 \mathrm{~mm} \mathrm{Hg}$ ). The remaining five patients had normal hemodynamics but they were receiving mechanical or inotropic assistance, or both. The hemodynamics in these six patients were first included in our analysis to provide an intentionto-treat evaluation. Then the results from these six patients were excluded to provide an efficacy analysis. Four patients had a perioperative myocardial infarction. In two of these four patients the highest CK-MB levels were less than $50 \mathrm{U} / \mathrm{L}$ and the CK-MB percent was less than $7 \%$ of the total CK level. The other two patients had higher levels of CK-MB. First, our evaluation of postoperative CK-MB level included all patients with an intention-to-treat analysis. Our second evaluation excluded those patients who had a myocardial infarction for an evaluation of. efficacy.

Cardioplegic solution flow rates and continuity. The initial antegrade cardioplegic solution infusion had similar flow rates among groups (antegrade cardioplegia, $310 \pm 18 \mathrm{ml} / \mathrm{min}$; retrograde cardioplegia, $325 \pm 22 \mathrm{ml} / \mathrm{min}$; combination cardioplegia, $331 \pm 23 \mathrm{ml} / \mathrm{min}$ ). The mean cardioplegic solution flow rate during continuous cardioplegia (excluding intermittent antegrade delivery of cardioplegic solution in the combination group) during aortic crossclamping was similar among groups (antegrade car- dioplegia, $144 \pm 11 \mathrm{ml} / \mathrm{min}$; retrograde cardioplegia, $163 \pm 13 \mathrm{ml} / \mathrm{min}$; combination cardioplegia, $147 \pm$ $10 \mathrm{ml} / \mathrm{min}$ ). Antegrade and retrograde infusions were intended to be provided at rates of $200 \mathrm{ml} / \mathrm{min}$ and "catch-up" infusions were intended to restore the $200 \mathrm{ml} / \mathrm{min}$ value. However, a careful stopwatch recording of the cardioplegic solution flow rates divided by the crossclamp time indicated that the actual infusions were less than $200 \mathrm{ml} / \mathrm{min}$. There were no significant differences among groups in the actual infusion rates as recorded by our observers. In the combination group, the infusion of $250 \mathrm{ml}$ intermittent antegrade cardioplegic solution was given $2.6 \pm 0.2$ times per case at a flow rate of $302 \pm$ $16 \mathrm{ml} / \mathrm{min}$. Cardioplegia was interrupted for a greater $(p<0.05)$ percentage of the crossclamp period during antegrade cardioplegia (antegrade cardioplegia, $36 \% \pm 4 \%$; combination cardioplegia, $17 \% \pm 3 \%$; retrograde cardioplegia, $28 \% \pm 4 \%$ ). The combination cardioplegia group received more cardioplegic solution (antegrade, $4.6 \pm 0.2 \mathrm{~L}$; combination, $6.3 \pm 0.4 \mathrm{~L}$; retrograde, $4.8 \pm 0.3 \mathrm{~L} ; p=$ 0.001 ).

Myocardial metabolism during cardioplegia. Myocardial oxygen consumption, lactate release, and acid release at 30 minutes and 50 minutes of crossclamping are shown in Fig. 2. Myocardial oxygen consumption was significantly $(p=0.0001$ by two-way analysis of variance) greater with antegrade cardioplegia and intermittent antegrade delivery of cardioplegic solution in combination cardioplegia than with retrograde cardioplegia or retrograde delivery of cardioplegic solution in combination cardioplegia, probably because coronary venous blood from the aortic root was contaminated with bronchial arterial blood. ${ }^{6}$ At 50 minutes of crossclamping, myocardial oxygen consumption was greatest with intermittent antegrade delivery of cardioplegic solution in the combination group than with either antegrade or retrograde cardioplegia, perhaps repaying an oxygen debt accumulated during continuous retrograde cardioplegia.

Myocardial lactate release increased with time during retrograde but not antegrade or combination cardioplegia and the interaction between cardioplegic group and time was significant $(p=0.01$ by two-way analysis of variance). Lactate release increased $(p<0.05)$ during retrograde but not during antegrade or combination cardioplegia by Duncan's test. Intermittent antegrade delivery of cardioplegic solution during combination cardioplegia resulted in greater $(p<0.05)$ lactate release at 30 minutes and 
50 minutes of crossclamping than antegrade or retrograde cardioplegia, which suggests the washout of lactate accumulated during continuous retrograde cardioplegia.

Myocardial acid release increased with time in the retrograde but not the antegrade cardioplegia group and the interaction between cardioplegic group and time was significant ( $p=0.02$ by two-way analysis of variance). A.cid release increased $(p<0.05)$ during retrograde cardioplegia by Duncan's test. Intermittent antegrade delivery of cardioplegic solution in combination cardioplegia had the greatest $(p<$ $0.05)$ acid release at 30 minutes and 50 minutes of crossclamping. Retrograde cardioplegia had greater $(p<0.05)$ acid release than antegrade cardioplegia at 50 minutes of crossclamping.

Myocardial metabolism during reperfusion. Myocardial oxygen extraction, lactate production, and acid production before crossclamping and during reperfusion are shown in Fig. 3. Myocardial oxygen extraction was greater after retrograde cardioplegia at the time of crossclamp removal and 10 minutes after discontinuation of CPB. Lactate production was found in all three groups immediately after crossclamp release and tended to be less after combination cardioplegia than after antegrade or retrograde cardioplegia; however, the differences were not statistically significant. Acid production tended to be greater after retrograde cardioplegia immediately after crossclamp removal and was significantly greater $(p<0.05)$ than that in antegrade cardioplegia 10 minutes after CPB.

Metabolic response to atrial pacing. The metabolic response to atrial pacing during and after $\mathrm{CPB}$ are summarized in Table III. Atrial pacing during CPB increased oxygen extraction $(p<0.05)$ compared with the baseline value after combination and retrograde cardioplegia but not after antegrade cardioplegia. Atrial pacing during CPB resulted in lactate production after antegrade and retrograde cardioplegia but in lactate extraction after combination cardioplegia. Atrial pacing during CPB tended to increase acid production compared with baseline values after antegrade $(p=0.06)$ and retrograde cardioplegia $(p=0.07)$ but not after combination cardioplegia $(p=0.35)$; however, the differences were not statistically significant. Ten minutes after discontinuation of $\mathrm{CPB}$ atrial pacing resulted in similarly small changes in oxygen extraction and lactate extraction after antegrade and combination cardioplegia but in lactate production after retrograde cardioplegia.

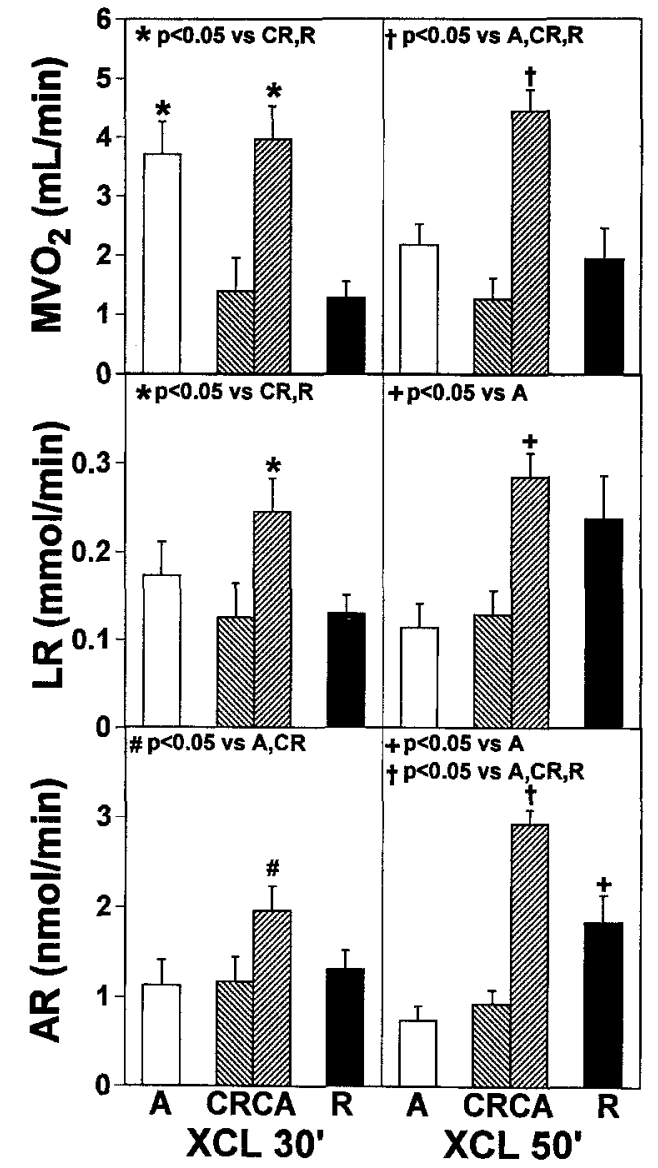

Fig. 2. Myocardial oxygen consumption $\left(\mathrm{MVO}_{2}\right)$, lactate release $(L R)$, and acid release $(A R)$ at 30 minutes $(X C L$ $\left.30^{\prime}\right)$ and 50 minutes $\left(X C L 50^{\prime}\right)$ of cardioplegic arrest are illustrated in three cardioplegic groups. Combination cardioplegic group is divided into antegrade and retrograde cardioplegic solution delivery. Myocardial oxygen consumption was greater with antegrade and intermittent antegrade delivery of cardioplegic solution in combination cardioplegia. Lactate release and acid release increased with time in retrograde cardioplegia but not in antegrade or combination cardioplegia. Intermittent antegrade delivery of cardioplegic solution in combination cardioplegia resulted in greatest lactate release and acid release during crossclamp period. $A$, Antegrade cardioplegia; $C A$, intermittent antegrade delivery of cardioplegic solution in combination group; $C R$, retrograde delivery of cardioplegic solution in combination group; $R$, retrograde cardioplegia.

Myocardial tissue lactate. Left ventricular tissue lactate concentrations increased immediately after antegrade cardioplegia but not after combination or retrograde cardioplegia $(p=0.004$ by analysis of 


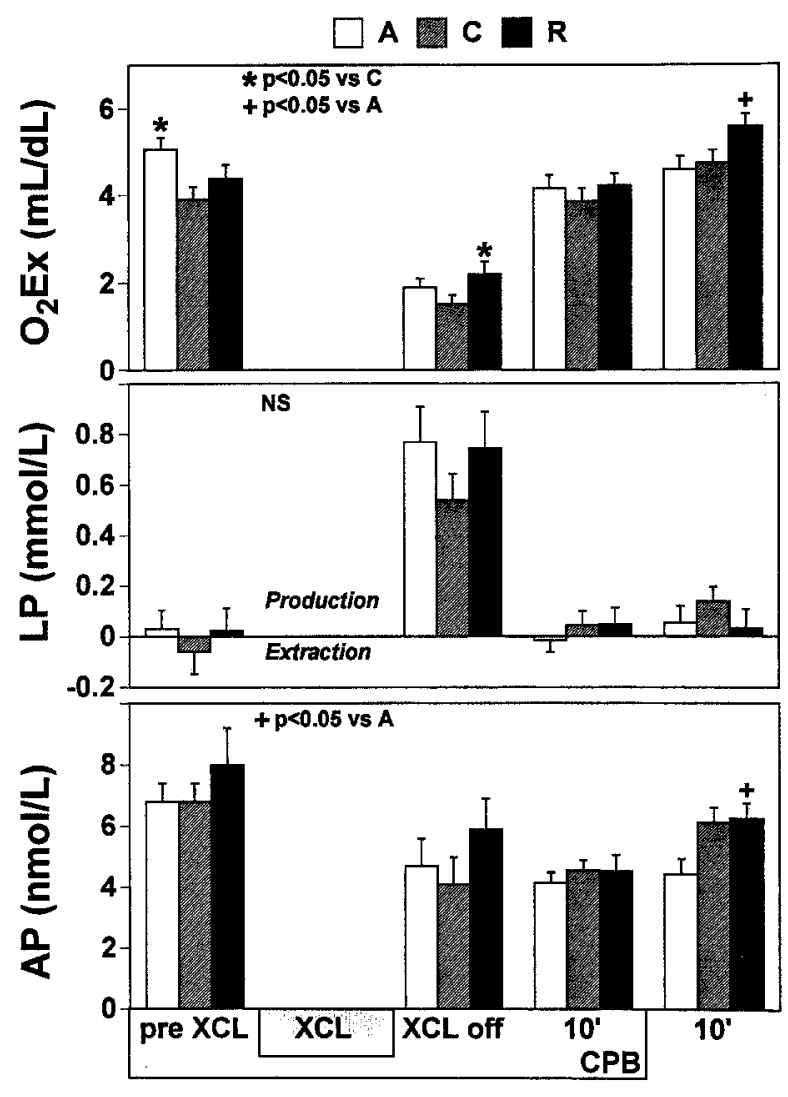

Fig. 3. Myocardial oxygen extraction $\left(\mathrm{O}_{2} \mathrm{Ex}\right)$, lactate production $(L P)$, and acid production $(A P)$ before crossclamp (pre $X C L)$, immediately after crossclamp removal (XCL off), 10 minutes after crossclamp removal (off $10^{\prime}$ ), and 10 minutes after cessation of $\mathrm{CPB}\left(\mathrm{CPB} 10^{\prime}\right)$. Myocardial oxygen extraction was greater after retrograde cardioplegia $(R)$. Lactate production was found in all three groups immediately after crossclamp release and tended to be less after combination cardioplegia $(C)$ than after antegrade $(A)$ or retrograde cardioplegia; however, differences were not statistically significant. Acid production tended to be greater after retrograde cardioplegia immediately after crossclamp removal and was significantly greater $(p<0.05)$ than that after antegrade cardioplegia 10 minutes after CPB. NS, Not significant.

variance, Fig. 4). Right ventricular lactate levels also increased immediately after crossclamp release $(p=$ 0.02 ) but there were no differences among cardioplegic groups.

Adenine nucleotides and degradation products. There were no differences between groups in the baseline levels of left ventricular ATP, ADP, AMP, or TDP (Table IV). Percentage change from baseline values is shown in Fig. 5. The decreases in ATP levels were greatest after retrograde cardioplegia
Table III. Metabolic response to atrial pacing (percent change)

\begin{tabular}{lccc}
\hline & Antegrade & Combination & Retrograde \\
\hline During CPB & & & \\
$\mathrm{O}_{2} \mathrm{Ex}$ & $63 \pm 47$ & $63 \pm 25^{*}$ & $20 \pm 7^{*}$ \\
$\mathrm{LP}$ & $36 \pm 58$ & $-22 \pm 197$ & $49 \pm 285$ \\
$\mathrm{AP}$ & $24 \pm 12$ & $14 \pm 14$ & $26 \pm 13$ \\
After CPB & & & \\
$\mathrm{O}_{2} \mathrm{Ex}$ & $7 \pm 6$ & $1 \pm 9$ & $-1 \pm 4$ \\
$\mathrm{LP}$ & $-42 \pm 50$ & $42 \pm 98$ & $-117 \pm 117^{*}$ \\
$\mathrm{AP}$ & $14 \pm 12$ & $12 \pm 8$ & $12 \pm 10$ \\
\hline
\end{tabular}

The results are presented as percentage change from baseline. $\mathrm{O}_{2} E x$, Oxygen extraction; $L P$, lactate production; $A P$, acid production; after $C P B$, 10 minutes after discontinuation of CPB.

${ }^{*} p<0.05$ atrial pacing different from baseline.

and least after combination cardioplegia at the time of crossclamp release $(p<0.05)$. There was no statistical difference in the decrease of ADP or AMP levels. TDP rose to a significantly higher level after retrograde cardioplegia than after antegrade or combination cardioplegia at the time of crossclamp removal $(p<0.05)$. Ten minutes after crossclamp release, ATP values were lowest after retrograde cardioplegia but the difference was not statistically significant $(p=0.10)$.

There were no significant differences between groups in baseline right ventricular values of ATP, ADP, AMP, and TDP (Table IV). At the time of crossclamp removal, there were no significant differences in ATP, ADP, and AMP levels among the three groups. However, TDP levels were significantly greater in the retrograde group than in the combination group. There were no differences in ATP, ADP, AMP, or TDP levels among groups after reperfusion (Table IV).

Hemodynamic measurements. Hemodynamic measurements are summarized in Tables $\mathrm{V}$ and VI. Before initiation of CPB afterload (mean arterial pressure) was lower and cardiac index was higher in the combination group but there were no differences in left ventricular stroke work index. There were no other differences among groups before CPB. Heart rate increased after operation in all groups: $66 \pm 2$ beats/min before initiation of CPB, $86 \pm 1$ beats/min 10 minutes after CPB, $86 \pm 2$ beats/min 1 hour after CPB, 87 \pm 1 beats/min 4 hours after CPB, $87 \pm 2$ beats/ $\min 12$ hours after $\mathrm{CPB}$, and $87 \pm 2$ beats $/ \mathrm{min} 24$ hours after CPB. Left ventricular stroke work index and cardiac index were greater $(p<0.05)$ after combination cardioplegia than after retrograde cardioplegia at 10 minutes after CPB de- 


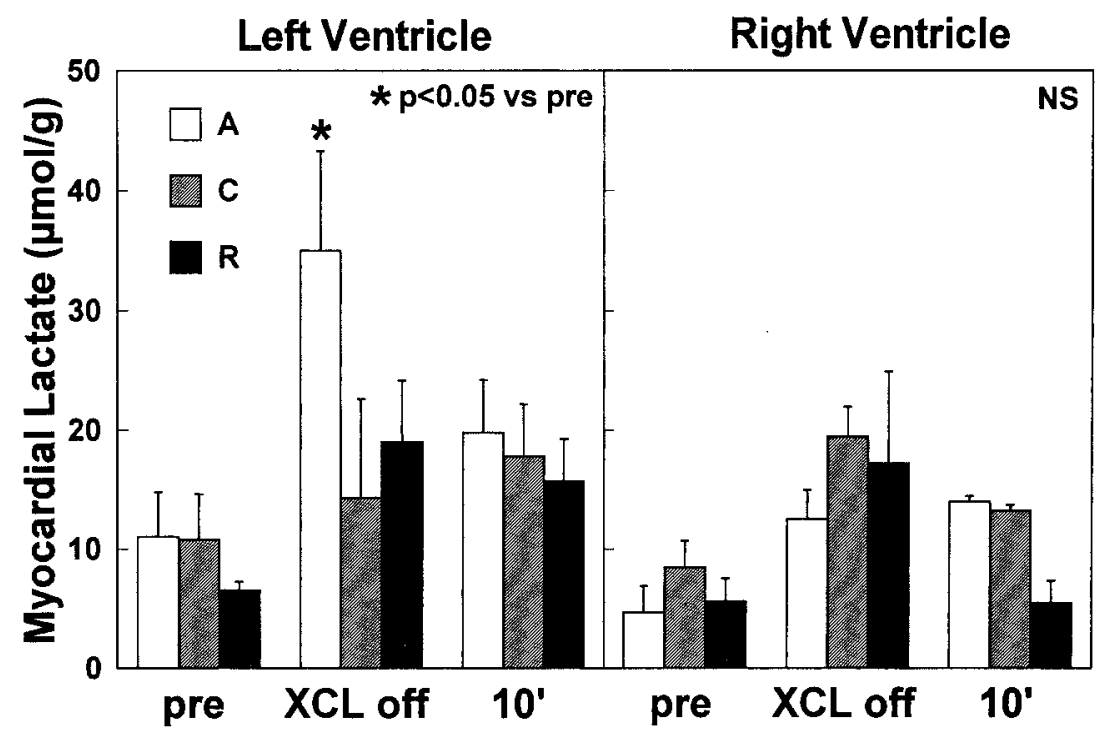

Fig. 4. Myocardial lactate concentrations in left and right ventricles before crossclamping (pre), at time of crossclamp removal (XCL off), and 10 minutes after crossclamp removal $\left(10^{\prime}\right)$ are illustrated. Left ventricular lactate concentrations increased immediately after antegrade $(A)$ but not after combination $(C)$ or retrograde $(R)$ cardioplegia $(p=0.004)$ by analysis of variance. Right ventricular lactate levels also increased immediately after crossclamp release $(p=0.02)$ but there were no differences between cardioplegic groups. NS, Not significant.

Table IV. Myocardial adenine nucleotides and adenine nucleotide degradation products

\begin{tabular}{|c|c|c|c|c|c|c|}
\hline & \multicolumn{3}{|c|}{ Left ventricle } & \multicolumn{3}{|c|}{ Right ventricle } \\
\hline & Antegrade & Combination & Retrograde & Antegrade & Combination & Retrograde \\
\hline \multicolumn{7}{|c|}{ ATP $(\mu \mathrm{mol} / \mathrm{gm}$ dry weight $)$} \\
\hline pre $\mathrm{XCL}$ & $16.9 \pm 1.3$ & $16.9 \pm 1.5$ & $19.2 \pm 2.9$ & $15.5 \pm 1.9$ & $11.9 \pm 3.4$ & $14.2 \pm 3.9$ \\
\hline $\mathrm{XCL}$ off & $12.7 \pm 0.9$ & $14.9 \pm 1.0^{*}$ & $10.7 \pm 1.0$ & $9.1 \pm 1.9$ & $11.8 \pm 1.1$ & $11.3 \pm 1.6$ \\
\hline XCL off $10^{\prime}$ & $13.7 \pm 1.3$ & $13.3 \pm 1.8$ & $12.8 \pm 1.6$ & $12.3 \pm 1.4$ & $11.9 \pm 1.2$ & $11.2 \pm 1.6$ \\
\hline \multicolumn{7}{|c|}{ ADP ( $\mu$ moll $/$ gm dry weight $)$} \\
\hline pre XCL & $2.06 \pm 0.25$ & $2.01 \pm 0.10$ & $1.96 \pm 0.45$ & $1.34 \pm 0.11$ & $2.37 \pm 1.09$ & $1.34 \pm 0.40$ \\
\hline $\mathrm{XCL}$ off & $2.42 \pm 0.35$ & $2.22 \pm 0.20$ & $1.86 \pm 0.29$ & $1.11 \pm 0.18$ & $1.64 \pm 0.21$ & $1.57 \pm 0.19$ \\
\hline $\mathrm{XCL}$ off $10^{\prime}$ & $1.92 \pm 0.17$ & $2.27 \pm 0.22$ & $2.37 \pm 0.19$ & $2.05 \pm 0.27$ & $1.96 \pm 0.25$ & $2.14 \pm 0.51$ \\
\hline \multicolumn{7}{|c|}{ AMP ( $\mu \mathrm{mol} / \mathrm{gm}$ dry weight) } \\
\hline pre $\mathrm{XCL}$ & $0.15 \pm 0.04$ & $0.14 \pm 0.01$ & $0.09 \pm 0.03$ & $0.09 \pm 0.03$ & $0.31 \pm 0.25$ & $0.07 \pm 0.04$ \\
\hline XCL off & $0.32 \pm 0.08$ & $0.20 \pm 0.02$ & $0.19 \pm 0.01$ & $0.13 \pm 0.01$ & $0.16 \pm 0.03$ & $0.14 \pm 0.02$ \\
\hline $\mathrm{XCL}$ off $10^{\prime}$ & $0.22 \pm 0.03$ & $0.24 \pm 0.04$ & $0.31 \pm 0.10$ & $0.45 \pm 0.20$ & $0.35 \pm 0.15$ & $0.41 \pm 0.16$ \\
\hline \multicolumn{7}{|c|}{ TDP ( $\mu \mathrm{mol} / \mathrm{gm}$ dry weight) } \\
\hline pre $\mathrm{XCL}$ & $0.33 \pm 0.07$ & $0.40 \pm 0.05$ & $0.19 \pm 0.14$ & $0.36 \pm 0.12$ & $0.34 \pm 0.12$ & $0.23 \pm 0.02$ \\
\hline $\mathrm{XCL}$ off & $0.83 \pm 0.15^{*}$ & $0.65 \pm 0.18^{*}$ & $1.85 \pm 0.16$ & $1.04 \pm 0.29$ & $0.67 \pm 0.12^{*}$ & $2.17 \pm 0.70$ \\
\hline $\mathrm{XCL}$ off $10^{\prime}$ & $0.50 \pm 0.14$ & $0.61 \pm 0.17$ & $0.45 \pm 0.04$ & $0.54 \pm 0.10$ & $0.54 \pm 0.05$ & $0.39 \pm 0.09$ \\
\hline
\end{tabular}

Values are given as mean plus or minus the standard error of the mean. pre XCL, Before crossclamp; XCL off, at the time of crossclamp removal; $X C L$ off $10^{\prime}, 10$ minutes after crossclamp removal.

${ }^{*} p<0.05$ versus retrograde group.

spite similar filling pressures and afterload (mean arterial pressure $)(p<0.05$ by analysis of covariance; Fig. 6 and Table V). The pulmonary capillary wedge pressure was greater $(p<0.05)$ after retrograde cardioplegia 24 hours after cessation of CPB (Table V). Right ventricular stroke work index was greater after combination cardioplegia than after retrograde cardioplegia 1 hour after 


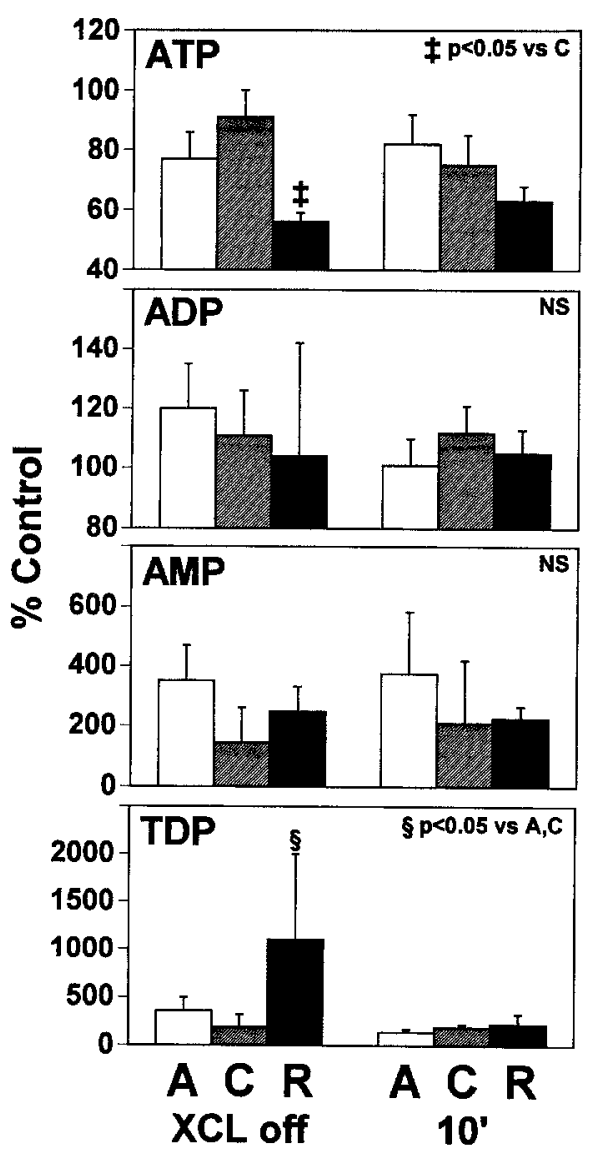

Fig. 5. Bar graph showing left ventricular ATP, ADP, AMP, and TDP concentrations at time of crossclamp removal (XCL off) and 10 minutes after crossclamp removal ( $X C L 10^{\prime}$ ) as percentages of baseline value. Decreases in ATP levels were greatest after retrograde cardioplegia $(R)$ and least after combination cardioplegia (C) at time of crossclamp release $(p<0.05)$. There was no statistical difference in decrease of ADP or AMP levels. TDP rose to significantly higher level after retrograde cardioplegia than after antegrade $(A)$ or combination cardioplegia at time of crossclamp removal $(p<0.05) . N S$, Not significant.

CPB despite a higher afterload (mean pulmonary artery pressure) and a similar filling pressure (right atrial pressure) (Fig. 7 and Table VI).

Excluding the six patients who had either inotropic support or intraaortic balloon pumping after operation did not change the conclusions or the statistical differences found when these patients were included. Among the 25 patients who received warm antegrade cardioplegia, one patient who required balloon pump support after operation had a higher left ventricular stroke work index and a lower pulmonary wedge pressure than the mean. Excluding the values of slightly increased stroke work index and lower pulmonary wedge pressure from this patient did not change the statistical evaluation. Among the 25 patients who received warm combination cardioplegia, three patients required either inotropic support ( 2 patients) or balloon pump support (1 patient). One patient (with a balloon pump) had a lower left ventricular stroke work index and a higher wedge pressure than the mean. Excluding these three patients did not change the mean cardiac index or stroke work index, which was greater after combination cardioplegia than after retrograde cardioplegia. Among the 25 patients who received warm retrograde cardioplegia, two patients required balloon pump support after operation. The left ventricular stroke work indices in these patients 10 minutes after discontinuation of CPB were 12 and $16 \mathrm{gm} \cdot \mathrm{m} / \mathrm{m}^{2}$ with pulmonary wedge pressures of 18 and $20 \mathrm{~mm} \mathrm{Hg}$, respectively. The exclusion of values from these two patients did not change the mean values, which were statistically significantly different $(p<0.05)$ from those of combination cardioplegia.

Postoperative release of CK-MB. There was no significant difference in total CK-MB release among groups (antegrade $1407 \pm 175$, combination $2018 \pm$ 394 , retrograde $2002 \pm 242 \mathrm{U} \times \mathrm{hr} / \mathrm{L}$ ). When the four patients with electrocardiographic changes were excluded, the CK-MB values were also not different among groups (antegrade $1407 \pm 175$, combination $1982 \pm 362$, retrograde $1982 \pm 231 \mathrm{U} \times \mathrm{hr} / \mathrm{L}$ ).

\section{Discussion}

Normothermic cardioplegia may allow resuscitation of the ischemic myocardium and immediate functional recovery in patients with high-risk conditions, reducing the morbidity and mortality. ${ }^{10,11}$ However, inadequate distribution of the solution or interruption of normothermic cardioplegic solution delivery may induce anaerobic metabolism and warm ischemic injury. Therefore delivery of the solution for normothermic cardioplegia must be continuous and homogeneous. To avoid ischemic myocardial damage during warm heart operations, improved techniques of cardioplegic solution delivery are required. $4,6,11$

This study was designed to evaluate the myocardial metabolic and ventricular functional response to different cardioplegic techniques. In these patients with low-risk conditions no differences in clinical outcomes were anticipated and none were 
Table V. Left ventricular hemodynamic measurements

\begin{tabular}{|c|c|c|c|c|c|c|}
\hline & Before $C P B$ & $10 \mathrm{~min}$ & $1 \mathrm{hr}$ & $4 h r$ & $12 \mathrm{hr}$ & $24 h r$ \\
\hline \multicolumn{7}{|c|}{$\operatorname{LVSWI~}\left(\mathrm{gm} \cdot \mathrm{m} / \mathrm{m}^{2}\right)$} \\
\hline A & $33.2 \pm 1.5$ & $25.3 \pm 1.7^{*}$ & $28.4 \pm 1.8$ & $32.0 \pm 1.5$ & $29.8 \pm 1.4$ & $30.4 \pm 1.1$ \\
\hline $\mathrm{C}$ & $33.6 \pm 2.3$ & $26.5 \pm 1.8^{*} \dagger$ & $26.6 \pm 1.7$ & $28.7 \pm 1.8$ & $27.8 \pm 1.5^{*}$ & $25.9 \pm 1.6^{*}$ \\
\hline $\mathrm{R}$ & $37.3 \pm 2.4$ & $20.8 \pm 1.4^{*}$ & $24.6 \pm 1.2^{*}$ & $28.6 \pm 1.9^{*}$ & $27.4 \pm 2.0^{*}$ & $28.5 \pm 1.7^{*}$ \\
\hline \multicolumn{7}{|c|}{ PCWP (mm Hg) } \\
\hline A & $12.8 \pm 1.0$ & $13.0 \pm 0.7$ & $11.1 \pm 0.6$ & $11.6 \pm 0.8$ & $12.5 \pm 0.7$ & $11.5 \pm 0.5$ \\
\hline $\mathrm{C}$ & $10.6 \pm 0.7$ & $14.5 \pm 0.8^{*}$ & $10.8 \pm 1.1$ & $11.1 \pm 0.6$ & $12.0 \pm 1.0$ & $12.6 \pm 0.9$ \\
\hline $\mathrm{R}$ & $11.4 \pm 0.6$ & $14.7 \pm 0.8^{*}$ & $10.0 \pm 0.7$ & $11.5 \pm 0.9$ & $13.8 \pm 1.0$ & $13.9 \pm 0.9^{*}+$ \\
\hline \multicolumn{7}{|c|}{ MAP (mm Hg) } \\
\hline A & $79.7 \pm 1.8$ & $65.5 \pm 1.5^{*}$ & $69.9 \pm 2.7^{*}$ & $77.0 \pm 2.3$ & $74.0 \pm 2.1$ & $73.8 \pm 1.8$ \\
\hline $\mathrm{C}$ & $71.9 \pm 2.9 \S$ & $65.3 \pm 2.0$ & $68.6 \pm 2.6$ & $73.5 \pm 2.4$ & $72.7 \pm 2.3$ & $70.5 \pm 2.6$ \\
\hline $\mathrm{R}$ & $80.8 \pm 2.3$ & $64.0 \pm 1.6^{*}$ & $69.4 \pm 1.2^{*}$ & $74.7 \pm 2.9^{*}$ & $70.2 \pm 2.0^{*}$ & $75.7 \pm 1.7^{*}$ \\
\hline \multicolumn{7}{|c|}{$\mathrm{CI}\left(\mathrm{L} / \mathrm{min} / \mathrm{m}^{2}\right)$} \\
\hline A & $2.3 \pm 0.1$ & $2.9 \pm 0.1^{*}$ & $2.9 \pm 0.2^{*}$ & $3.0 \pm 0.1^{*}$ & $3.0 \pm 0.1^{*}$ & $3.0 \pm 0.1^{*}$ \\
\hline $\mathrm{C}$ & $2.7 \pm 0.1+$ & $3.2 \pm 0.1^{*} \dagger$ & $2.9 \pm 0.1 \dagger$ & $3.0 \pm 0.1$ & $3.0 \pm 0.1$ & $2.8 \pm 0.1$ \\
\hline $\mathrm{R}$ & $2.4 \pm 0.1$ & $2.7 \pm 0.1$ & $2.6 \pm 0.1$ & $2.9 \pm 0.2^{*}$ & $2.9 \pm 0.1^{*}$ & $2.9 \pm 0.1^{*}$ \\
\hline
\end{tabular}

Values are given as mean plus or minus the standard error of the mean. pre, Before CPB; $10 \mathrm{~min}, 10$ minutes after CPB; $1 \mathrm{hr}, 1$ hour after CPB; $4 \mathrm{hr}, 4 \mathrm{hours}$ after CPB; $12 \mathrm{hr}, 12$ hours after CPB; $24 \mathrm{hr}, 24$ hours after CPB; $L V S W$, left ventricular stroke work index; $A$, antegrade cardioplegia; $C$, combination cardioplegia; $R$, retrograde cardioplegia; $P C W P$, pulmonary capillary wedge pressure; $M A P$, mean arterial pressure; $C I$, cardiac index.

${ }^{*} p<0.05$ versus before CPB.

$\uparrow p<0.05$ versus retrograde cardioplegia.

$\stackrel{t}{p}<0.05$ versus antegrade cardioplegia.

$\$ p<0.05$ versus antegrade and retrograde cardioplegia.

Table VI. Right ventricular hemodynamic measurements

\begin{tabular}{|c|c|c|c|c|c|c|}
\hline & Before $C P B$ & $10 \mathrm{~min}$ & $l \mathrm{hr}$ & $4 h r$ & $12 \mathrm{hr}$ & $24 h r$ \\
\hline \multicolumn{7}{|c|}{ RVSWI $\left(\mathrm{gm} \cdot \mathrm{m} / \mathrm{m}^{2}\right)$} \\
\hline $\mathrm{A}$ & $3.7 \pm 0.3$ & $4.1 \pm 0.6$ & $2.9 \pm 0.4$ & $3.8 \pm 0.4$ & $3.9 \pm 0.3$ & $3.6 \pm 0.3$ \\
\hline $\mathrm{C}$ & $4.3 \pm 0.4$ & $4.7 \pm 0.5$ & $3.6 \pm 0.4 *$ & $2.9 \pm 0.3 \dagger$ & $3.5 \pm 0.3$ & $3.5 \pm 0.3$ \\
\hline $\mathrm{R}$ & $3.6 \pm 0.5$ & $3.6 \pm 0.4$ & $1.9 \pm 0.2 \dagger$ & $3.2 \pm 0.4$ & $3.7 \pm 0.4$ & $3.6 \pm 0.2$ \\
\hline \multicolumn{7}{|c|}{$\mathrm{RAP}(\mathrm{mm} \mathrm{Hg})$} \\
\hline A & $9.2 \pm 0.6$ & $10.0 \pm 0.8$ & $9.3 \pm 0.7$ & $10.2 \pm 0.7$ & $9.5 \pm 0.7$ & $10.0 \pm 0.8$ \\
\hline $\mathrm{C}$ & $8.0 \pm 0.5$ & $10.9 \pm 0.6 \dagger$ & $9.8 \pm 0.6$ & $9.3 \pm 0.6$ & $9.4 \pm 0.6$ & $9.6 \pm 0.7$ \\
\hline $\mathrm{R}$ & $8.8 \pm 0.7$ & $11.0 \pm 0.8$ & $9.8 \pm 0.9$ & $9.0 \pm 0.6$ & $10.3 \pm 0.9$ & $10.8 \pm 0.9$ \\
\hline \multicolumn{7}{|c|}{ MAP (mm Hg) } \\
\hline A & $16.7 \pm 0.9$ & $18.3 \pm 0.8$ & $15.2 \pm 0.6$ & $17.9 \pm 0.9$ & $17.4 \pm 0.9$ & $17.3 \pm 0.8$ \\
\hline $\mathrm{C}$ & $15.8 \pm 1.0$ & $20.0 \pm 1.0 \dagger$ & $17.6 \pm 1.1^{*}$ & $15.7 \pm 0.7$ & $17.5 \pm 1.1$ & $17.7 \pm 1.0$ \\
\hline $\mathrm{R}$ & $15.6 \pm 0.7$ & $19.1 \pm 0.9 \dagger$ & $14.4 \pm 0.8$ & $16.3 \pm 1.1$ & $18.0 \pm 1.0$ & $18.3 \pm 1.1$ \\
\hline
\end{tabular}

Values are given as mean plus or minus the standard error of the mean. RVSWI, Right ventricular stroke work index; $R A P$, right atrial pressure; $M P A$, mean pulmonary artery pressure. Other abbreviations as in Table V.

" $p<0.05$ versus retrograde.

$\grave{\dagger} p<0.05$ versus before CPB.

found. Because we found differences in the metabolic and hemodynamic response to elective cardiac operations, we plan to perform a prospective, randomized trial in patients with high-risk conditions. The patients were first evaluated by an intention-totreat process. Differences were discovered in both myocardial metabolism and ventricular function. However, patients who require inotropic or balloon pump support have postoperative hemodynamics that are not dependent on the cardioplegic techniques. Therefore analysis was also done with the exclusion of the six patients in whom these therapies were used, and significant differences were maintained among groups. The inclusion of patients who received inotropic support does not permit an assessment of ventricular function independent of preload or afterload. No significant differences were found among groups in the amount of CK-MB released after operation. The analysis was done first by an intention-to-treat process and then for efficacy by excluding the four patients who had electrocardiographic changes. Neither analysis found signifi- 


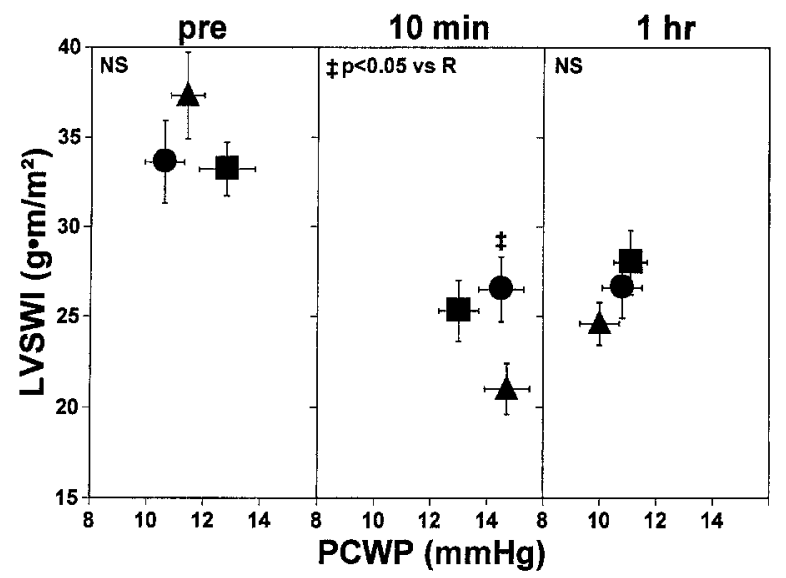

Fig. 6. Relationships between left ventricular stroke work index (LVSWI) and pulmonary capillary wedge pressure (PCWP) before CPB (pre) and 10 minutes (10 min) and 1 hour $(1 \mathrm{hr})$ after CPB are depicted. Left ventricular stroke work index was greatest after combination cardioplegia (circles) and least after retrograde cardioplegia (triangles; $R$ ) despite similar filling pressure (pulmonary capillary wedge pressure) 10 minutes after cessation of CPB ( $p<0.05$ by analysis of covariance). Squares represent values for antegrade cardioplegia; $N S$, not significant.

cant differences among groups. The primary analysis in this study was the intention-to-treat analysis and the secondary analysis was the efficacy evaluation of various cardioplegic techniques. The perioperative measurements of the three patients who died after operation were included in this evaluation because the deaths occurred more than 72 hours after the operation.

Antegrade cardioplegia. In previous studies we found that warm antegrade delivery of cardioplegic solution improved myocardial metabolic recovery, increased early postoperative end systolic elastance and preload recruitable stroke work, and improved early diastolic relaxation compared with results with cold antegrade delivery of cardioplegic solution. ${ }^{1,12}$ However, animal studies demonstrated that nutritive flow to the myocardium supplied by an occluded left anterior descending coronary artery was substantially reduced with antegrade delivery of cardioplegic solution, particularly in the subendocardium. $^{2}$ In the setting of acute left anterior descending coronary occlusion postischemic global and regional systolic function was depressed after warm antegrade cardioplegic solution delivery compared with that after warm retrograde cardioplegic solution delivery. ${ }^{11}$ Furthermore, the anterior myo-

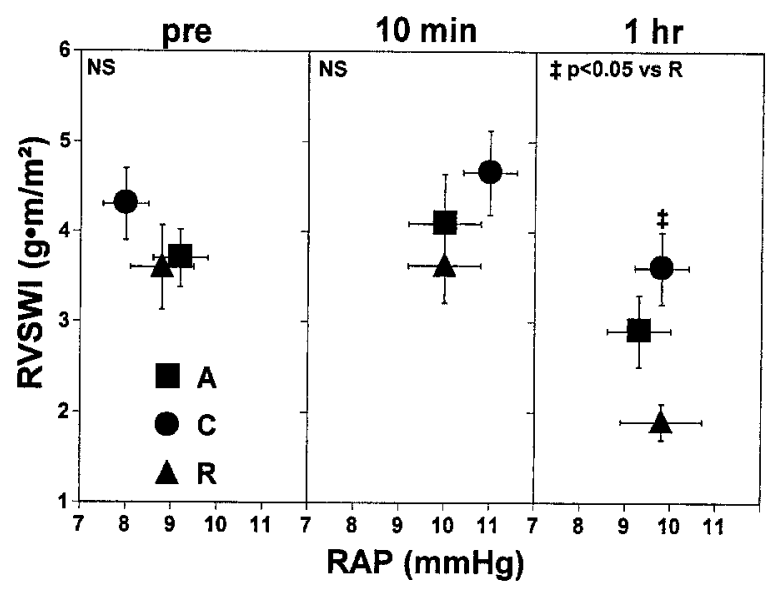

Fig. 7. Relationships between right ventricular stroke work index (RVSWT) and right atrial pressure (RAP) before CPB (pre) and 10 minutes $(10 \mathrm{~min})$ and 1 hour (1 hr) after CPB are depicted. Right ventricular stroke work index was greatest after combination cardioplegia $(C)$ and least after retrograde cardioplegia $(R)$ despite similar filling pressure (right atrial pressure) at 1 hour after CPB. NS, Not significant; $A$, antegrade cardioplegia.

cardium may not be perfused during antegrade cardioplegic solution delivery when left anterior descending coronary artery revascularization with an internal mammary artery graft precludes vein graft infusions. In the present study myocardial lactate concentrations increased significantly in the territory of the left anterior descending coronary artery after antegrade cardioplegia but not after combination or retrograde cardioplegia. This lactate increase is the first evidence in human beings that warm antegrade delivery of cardioplegic solution does not adequately perfuse the territory of the left anterior descending artery when a mammary artery graft is used.

Retrograde cardioplegia. Retrograde delivery of coronary sinus cardioplegic solution has been proposed to be a superior technique of myocardial protection because it permits delivery of cardioplegic solution beyond coronary obstructions or critical coronary stenoses. ${ }^{2,13-15}$ However, shunting of cardioplegic solution administered in a retrograde fashion away from nutrient capillaries into venous sinusoids and thebesian channels may limit cardioplegic solution distribution. ${ }^{3,}{ }^{16}$ In a recent study we found that warm retrograde delivery of cardioplegic solution results in decreasing anaerobic lactate production when flow rates are increased from 50 to 200 $\mathrm{ml} / \mathrm{min} .{ }^{6}$ However, further increases to 300 or 500 $\mathrm{ml} / \mathrm{min}$ increased the shunt flow without increasing 
myocardial oxygen extraction and without reducing anaerobic lactate production. Further, cardioplegic solution administered in a retrograde fashion may inadequately perfuse the right ventricle and posterior left ventricle or posterior septum. ${ }^{2,3,15,16} \mathrm{We}$ also demonstrated that warm retrograde delivery of cardioplegic solution resulted in greater anaerobic lactate production during and after cardioplegic arrest compared with results with other cardioplegic techniques. ${ }^{5,17,18}$ In this study lactate and acid release increased with time during retrograde cardioplegia but not during antegrade or combination cardioplegia. In addition, myocardial lactate levels were higher than baseline values in the right but not the left ventricle, which suggests incomplete right ventricular protection. Significant decreases in ATP levels in the left ventricle and significant accumulations in TDP levels in both left and right ventricles after retrograde cardioplegia suggest that inadequate cardioplegic solution delivery resulted in ATP metabolism to diffusible precursors that were not washed out by retrograde delivery of cardioplegic solution. ${ }^{7}$ The depletion of ATP, accumulation of TDP, and increased lactate and acid release are consistent with more severe myocardial ischemia during retrograde cardioplegic solution delivery. The depressed metabolism may have contributed to the depressed left and right ventricular function in the early postoperative period. Warm retrograde delivery of cardioplegic solution did not provide optimal myocardial protection for either the right ventricle or left ventricle.

Combination cardioplegia. In a previous animal study that used radiolabeled microspheres, the distribution of cardioplegic solution administered in an antegrade fashion was significantly different from that of cardioplegic solution administered in a retrograde fashion. ${ }^{19}$ To overcome this disparity, intermittent antegrade and then retrograde cold cardioplegic solutions were given intermittently in animals $^{20}$ and patients. ${ }^{21,22}$ Intermittent infusions of antegrade and then retrograde cold blood cardioplegic solutions every 20 minutes provided homogeneous myocardial cooling, complete left and right ventricular functional recovery in animals, and excellent clinical outcomes in patients. However, this cardioplegic technique cannot be applied to warm heart operations. Therefore we developed the technique of continuous retrograde delivery of cardioplegic solution with intermittent antegrade infusions.

During combination cardioplegia, intermittent antegrade infusions resulted in an increase in myo- cardial oxygen consumption and lactate and acid release, which suggests a washout of anaerobic metabolites and repayment of the oxygen debt accumulated during retrograde cardioplegic solution infusion. Combination cardioplegia prevented the progressive increase in lactate and acid release during the crossclamp period that is seen with retrograde cardioplegia. After crossclamp release lactate and acid production were least after combination cardioplegia, and postoperative atrial pacing did not induce lactate production, which suggests better myocardial protection with this technique. Combination cardioplegia best preserved ATP levels and prevented the accumulation of myocardial lactate in the left but not the right ventricle. Both left and right ventricular function were better after combination cardioplegia.

In summary, intermittent antegrade infusions of cardioplegic solution during continuous warm retrograde cardioplegic solution delivery provided homogeneous distribution and prevented ischemic myocardial injury during warm heart operations.

\section{REFERENCES}

1. Yau TM, Weisel RD, Mickle DAG, et al. Optimal delivery of blood cardioplegia. Circulation 1991; 84(Suppl):III380-8.

2. Partington MT, Acar C, Buckberg GD, Julia P, Kofsky ER, Bugyi HI. Studies of retrograde cardioplegia: I-capillary blood flow distribution to myocardium supplied by open and occluded arteries. J THORAC CARdiovasc Surg 1989;97:605-12.

3. Caldarone CA, Krukenkamp IB, Misare BD, Levitsky $S$. Perfusion deficits with retrograde warm blood cardioplegia. Ann Thorac Surg 1994;57:403-6.

4. Fremes SE, Christakis GT, Weisel RD, et al. A clinical trial of blood and crystalloid cardioplegia. J Thorac Cardiovasc Surg 1984;88:726-41.

5. Christakis GT, Fremes SE, Weisel RD, et al. Reducing the risk of urgent revascularization for unstable angina: a randomized clinical trial. J Vasc Surg 1986; 3:764-72.

6. Ikonomidis JS, Yau TM, Weisel RD, et al. Optimal flow rates for retrograde warm cardioplegia. J THoRAC CARDIOvasC SuRg 1994;107:510-9.

7. Yau TM, Weisel RD, Mickle DA, et al. Alternative techniques of cardioplegia. Circulation 1992;86(Suppl): II377-84.

8. Hull-Ryde EA, Lewis WR, Veronee CD, Lowe JE. Simple step gradient elution of the major high-energy compounds and their catabolites in cardiac muscle using high-performance liquid chromatography. J Chromatogr 1986;377:165-74.

9. Harris RC, Hultman E, Nordesjo L-O. Glycogen, 
glycolytic intermediates and high-energy phosphates determined in biopsy samples of musculus quadriceps femoris of man at rest: methods and variance of values. Scand J Clin Lab Invest 1974; 33:109-20.

10. Lichtenstein SV, Abel JG, Salerno TA. Warm heart surgery and results of operation for recent myocardial infarction. Ann Thorac Surg 1991;52:455-60.

11. Vaughn CC, Opie JC, Florendo FT, Lowell PA, Austin J. Warm blood cardioplegia. Ann Thorac Surg 1993;55:1227-32.

12. Yau TM, Ikonomidis JS, Weisel RD, et al. Ventricular function after normothermic versus hypothermic cardioplegia. J Thorac Cardiovasc Surg 1993;105: 833-44.

13. Misare BD, Krukenkamp IB, Lazer ZP, Levitsky S. Retrograde is superior to antegrade continuous warm blood cardioplegia for acute cardiac ischemia. Circulation 1992;86(Suppl):II393-7.

14. Menasche P, Piwnica A. Cardioplegia by the way of the coronary sinus for valvular and coronary surgery. J Am Coll Cardiol 1991;18:628-36.

15. Aronson S, Lee BK, Liddicoat JR, et al. Assessment of retrograde cardioplegia distribution using contrast echocardiography. Ann Thorac Surg 1991;52:810-4.

16. Solorzano J, Taitelbaum G, Chiu CJ. Retrograde coronary sinus perfusion for myocardial protection during cardiopulmonary bypass. Ann Thorac Surg 1978;25:201-8.

17. Yau TM, Ikonomidis JS, Weisel RD, et al. Which techniques of cardioplegia prevent ischemia? Ann Thorac Surg 1993;56:1020-8.

18. Hayashida N, Ikonomidis JS, Weisel RD, et al. The optimal cardioplegic temperature. Ann Thorac Surg 1994;58:961-71.

19. Aldea GS, Hou D, Fonger JD, Shemin RJ. Inhomogeneous and complementary antegrade and retrograde delivery of cardioplegic solution in the absence of coronary artery obstruction. J THORAC CARDIOVASC Surg 1994;107:499-504.

20. Partington MT, Acar C, Buckberg GD, Julia PL. Studies of retrograde cardioplegia: II-advantages of antegrade/retrograde cardioplegia to optimize distribution in jeopardized myocardium. J THORAC CARDIOVASC SURG 1989;97:613-22.

21. Buckberg GD, Drinkwater DC, Laks H. A new technique for delivering antegrade/retrograde blood cardioplegia without right heart isolation. Eur J Cardiothorac Surg 1990;4:163-8.

22. Drinkwater DC, Cushen CK, Laks H, Buckberg GD. The use of combined antegrade-retrograde infusion of blood cardioplegic solution in pediatric patients undergoing heart operation. $J$ THORAC CARDIOvaSC SURG 1992;104:1349-55. 